\title{
Las redes sociales en la estrategia de implementación de evidencias en la práctica clínica: experiencia del Hospital Regional Universitario de Málaga, España
}

Social networks in the clinical practice evidence implementation strategy: experience from Hospital Regional Universitario de Málaga, Spain.

Redes sociais na estratégia de implementação de evidências na prática clínica: experiência do Hospital Regional Universitario de Málaga, Espanha

Jesús Bujalance-Hoyos, Enf. ${ }^{1} \oplus$, Doris Grinspun, Enf., MSc., PhD. ${ }^{2} \oplus$, María Teresa Pérez-Jiménez, Enf. ${ }^{3}{ }^{\circledR}$, Cipriano Viñas-Vera, Enf., PhD. ${ }^{4} \oplus$, María Soledad Jiménez-Fernández, Enf. Lic. ${ }^{5}$ (1), Juan Antonio García-Sánchez, Enf. Esp. ${ }^{6}$ (])

1. Enfermero. Instituto de Investigación Biomédica de Málaga - IBIMA. Área de Procesos de Cuidados de Enfermería, Hospital Regional Universitario de Málaga, España.

2. Registered Nurse, Master of Science in Nursing, doctor of Philosophy, doctor of Law (hon), doctor Honouris Causa, gerente general, Asociación de Enfermeras Profesionales, Ontario, Canadá (RNAO).

3. Enfermera. Unidad de Salud Mental, Hospital Regional Universitario de Málaga, España.

4. Enfermero, Doctor en Ciencias de la Salud. Instituto de Investigación Biomédica de Málaga - IBIMA. Málaga, España.

5. Enfermera, Licenciada en Administración y Dirección de Empresas. Instituto de Investigación Biomédica - IBIMA. Hospital Universitario Regional de Málaga, España.

6. Enfermero, Especialista en Enfermería Psiquiátrica. Coordinador de Cuidados de la UGC de Salud Mental del Hospital Regional Universitario de Málaga, España.

Correspondencia. Jesús Bujalance Hoyos. Instituto de Investigación Biomédica de Málaga - IBIMA. Área de Procesos de Cuidados de Enfermería, Hospital Regional Universitario de Málaga. Tel. +34 671568295. Email. jesus.bujalance.sspa@juntadeandalucia.es

Cómo citar: Bujalance Hoyos J, Grinspun D, Pérez Jiménez MT, Viñas Vera C, Jiménez Fernández MS, García Sánchez JA. Las redes sociales en la estrategia de implementación de evidencias en la práctica clínica: experiencia del Hospital Regional Universitario de Málaga, España. MedUNAB. 2020;23(1):107-117. doi:10.29375/01237047.3571

\section{INFORMACIÓN DEL ARTÍCULO:}

Artículo recibido: 20 de marzo de 2019

Artículo aceptado: 06 de febrero de 2020

DOI: https://doi.org/10.29375/01237047.3571 


\section{RESUMEN}

Introducción. Twitter se ha convertido en el foro favorito para la comunicación de la atención médica, en cuanto permite a los usuarios publicar y compartir mensajes fácilmente a sus seguidores. En el Hospital Regional Universitario de Málaga (HRUM) se ha recurrido al uso de las redes sociales, particularmente Twitter, para facilitar la implementación y difusión de las recomendaciones de las Guías de Buenas Prácticas (GBP) de la Registered Nurses Association of Ontario (RNAO) en la práctica clínica. El objetivo del presente artículo es describir la estrategia y reflexionar acerca del rol de las redes sociales en las estrategias y los resultados de implantación de recomendaciones de GBP de la RNAO. Temas de reflexión. Siguiendo la metodología del modelo Best Practice Spotlight Organization (BPSO), se ha otorgado un papel protagonista a las enfermeras asistenciales de cada unidad a través de la formación, creación de equipos de implantación y difusión del programa. El uso de nuevos registros y, sobre todo, el uso de las redes sociales, ha obtenido unos resultados excelentes de adherencia de los profesionales al programa tanto a nivel cuantitativo como cualitativo. Conclusiones. El uso de las redes sociales como estrategia de difusión en la implantación de las recomendaciones de las GBP de la RNAO ha conseguido muy buena acogida por parte de los profesionales, pues muestra un alto nivel de participación, y es una herramienta útil como estrategia de difusión. Se necesita más tiempo para monitorizar el uso de las redes sociales y su posible impacto en la implantación de evidencias y la mejora de los resultados de salud de los pacientes y organizaciones de salud.

Palabras claves:

Guía de Práctica Clínica; Práctica Clínica Basada en la Evidencia; Enfermería Basada en la Evidencia; Comunicación; Medios de Comunicación; Red Social.

\section{ABSTRACT}

Introduction. Twitter has become the favored forum for communicating health care, since it allows users to publish and share messages with their followers. At Hospital Regional Universitario de Málaga (HRUM), they have used social networks, particularly Twitter, to facilitate implementing and broadcasting the recommendations from the Registered Nurses Association of Ontario's (RNAO) Best Practice Guideline (BPG) for clinical practice. The objective of this paper is to describe the strategy and reflect on the role of social networks on the strategies and results of implementing the RNAO's BPG recommendations. Topics of reflection. In accordance to the methodology of the Best Practice Spotlight Organization (BPSO) model, a leading role has been given to the nurses at each unit through training, creation of implementation teams and broadcasting the program. Using new records and, most of all, social networks, the strategy has obtained excellent results from professionals when it comes to adherence to the program, both quantitatively and qualitatively. Conclusions. The use of social networks as a broadcasting strategy in implementing the RNAO's BPG recommendations has been well received by professionals. The strategy shows high participation levels and is a useful tool as a broadcasting strategy. More time is needed to monitor social network use and its possible impact on generating evidence, as well as the improvement of healthcare organizations and patient's health results.

Keywords:

Practice Guideline; Evidence-based Practice; Evidence-based Nursing; Communication; Communications Media; Social Networking.

\section{RESUMO}

Introdução. O Twitter se tornou o fórum favorito para a comunicação no atendimento médico, pois permite que os usuários publiquem e compartilhem facilmente mensagens para seus seguidores. No Hospital Regional Universitário de Málaga (HRUM), foram utilizadas as redes sociais, particularmente o Twitter, para facilitar a implementação e disseminação das recomendações das Guias de Boas Práticas (GBP) da Registered Nurses Association of Ontario (RNAO) na prática clínica. O objetivo deste artigo é descrever a estratégia e refletir sobre o papel das redes sociais nas estratégias e nos resultados da implementação das recomendações do GBPs da RNAO. Tópicos de reflexão. Seguindo a metodologia 
do modelo Best Practice Spotlight Organization, foi atribuído um papel essencial às enfermeiras de cada unidade por meio de treinamento, criação de equipes de implementação e divulgação do programa. O uso de novos registros e, sobretudo, o uso de redes sociais, obteve excelentes resultados de adesão dos profissionais ao programa, tanto quantitativa quanto qualitativamente. Conclusões. O uso das redes sociais como estratégia de disseminação na implementação das recomendações do GBPs da RNAO alcançou uma boa recepção pelos profissionais, pois mostra um alto nível de participação e é uma ferramenta útil como estratégia de disseminação. É necessário mais tempo para monitorar o uso das redes sociais e seu possível impacto na implementação de evidências e na melhoria dos resultados de saúde de pacientes e organizações de saúde.

Palavras-chave:

Guia de Prática Clínica; Prática Clínica Baseada em Evidências; Enfermagem Baseada em Evidências; Comunicação; Meios de comunicação; Rede social.

\section{Introducción}

Las redes sociales se han convertido en un método de comunicación establecido en nuestra sociedad actual (1). El uso de la tecnología digital muestra un crecimiento rápido y extendido en los últimos años. En Estados Unidos, por ejemplo, se estima que $95 \%$ de los habitantes usan teléfonos móviles; $89 \%$, internet; $77 \%$, smartphone, y $69 \%$, redes sociales (2).

La red social de mayor uso por los americanos es Youtube (73\%), seguida de Facebook (68\%), Instagram (35\%) y Twitter (24\%) (3). La importancia del uso de las redes sociales va en aumento y muchos organismos oficiales, instituciones y gobiernos, hacen públicas sus opiniones o informaciones a través de las mismas.

Twitter se ha convertido en el foro favorito para la comunicación de atención médica para médicos, defensores de pacientes y organizaciones de atención médica. Por medio de Twitter, un usuario puede publicar mensajes ("tweets") de hasta 280 caracteres, y replicar mensajes publicados por otros usuarios. El uso de un hashtag (una palabra o frase precedida por el signo \#, como \#breastcancer o \#myeloma) en un tweet sirve para vincular el mensaje con una conversación o una comunidad virtual, con el propósito de que se pueda buscar fácilmente información sobre este tema en Twitter (1).

Por otra parte, el Programa de Implantación de Buenas Prácticas en Cuidados, "Best Practice Spotlight Organization (BPSO)", basado en las Guías Clínicas RNAO (Registered Nurses Association of Ontario), surge en España del acuerdo firmado en 2012 entre la Unidad de Investigación en Cuidados de Salud (Investén-isciii) del Instituto de Salud Carlos III, y la RNAO. Por este acuerdo, Investén-isciii se constituye en el Host o Centro Coordinador del programa BPSO de RNAO en España (4), y este programa se tradujo al castellano como Centros Comprometidos con la Excelencia en Cuidados (CCEC).

Para llevar a cabo esta tarea, se utilizan las Guías de Buenas Prácticas (GBP) desarrolladas por la RNAO (5) y su herramienta de implantación (6), que identifica seis fases para poder llevar a cabo la implantación de una GBPs: identificación del problema y selección de la evidencia, adaptación al contexto, valoración de barreras y facilitadores, selección de intervenciones a implantar, monitorización y evaluación y sostenibilidad.

En 2017, tras ser seleccionado en una convocatoria pública a nivel nacional en España, el Hospital Regional Universitario de Málaga se incorporó a este programa, convirtiéndose así en centro candidato al programa "Centros Comprometidos con la Excelencia en Cuidados" (CCEC), con el objetivo de implantar cinco GBP de la RNAO en un periodo de tres años: cuidados y mantenimiento de los accesos vasculares para reducir las complicaciones (7), prevención de caídas y disminución de las lesiones derivadas de las caídas (8), cuidados y manejo de la ostomía (9), valoración y manejo de las úlceras de pie diabético (10), y valoración y cuidados de los adultos en riesgo de ideación y comportamiento suicida (11). Este programa pretende mejorar la práctica clínica de enfermería, los resultados de salud de los pacientes, y los resultados de efectividad de las instituciones sanitarias (12-14).

Dichas iniciativas se sustentan en que la práctica clínica basada en la evidencia mejora los resultados clínicos $(15,16)$, pero existe una brecha entre el conocimiento que se genera y la práctica profesional (17). Diversos estudios concluyen que aproximadamente el $30-40 \%$ de los pacientes no reciben una atención según la evidencia científica actual, y que alrededor del $20-25 \%$ de la atención que se proporciona es innecesaria e inclusive puede resultar peligrosa (18). 
Por lo tanto, en 2018 Investén-isciii constituyó su tercera cohorte de CCEC, en la que participan ocho instituciones sanitarias españolas, y durante su fase de implantación de recomendaciones, sobresalió el uso de redes sociales -en particular Twitter- como una estrategia importante para compartir conocimientos y motivar la transferencia de evidencia hacia la práctica clínica. Dada la realidad de una sociedad conectada virtualmente, el uso responsable de redes sociales puede resultar beneficioso.

El cuidado de la salud no es ajeno a la evolución de las redes sociales: actualmente, más del $40 \%$ de los consumidores de atención médica utilizan estos medios para sus necesidades de información de atención médica (19). Los hospitales y los profesionales de la salud se relacionan entre sí por medio de las redes sociales en sus campos de trabajo. El $41 \%$ de los consumidores de atención de salud eligieron las redes sociales para decidir sobre sus proveedores de atención. Además, el 26\% de los hospitales de Estados Unidos participa actualmente de alguna forma en las redes sociales (20).

Conociendo la importancia de las redes entre los profesionales sanitarios, se ha querido introducir esta herramienta en la ecuación de nuestra estrategia de implantación de recomendaciones dentro del programa CCEC. Por este motivo, se propone en este artículo describir la experiencia de implantación de las guías con diferentes estrategias, y reflexionar en torno a la importancia del uso de las redes sociales para implantar las recomendaciones de las GBP de la RNAO en el HRUM entre los profesionales, instituciones y partes interesadas.

\section{Metodología de implementación de la estrategia}

La RNAO desarrolló la herramienta de implantación de buenas prácticas para ayudar a los profesionales en la transferencia de la evidencia de sus GBP a la práctica clínica (6). Esta implantación comprende diferentes actividades, entre las que se incluyen: la creación de estructuras organizativas que den soporte a los grupos de trabajo, la difusión de la iniciativa en el centro y la captación y formación de impulsores que son los profesionales "líderes en pijama" o "champions", como los denomina la RNAO, quienes adquieren el compromiso de facilitar el desarrollo de la implantación de las recomendaciones de las GBP junto a los colaboradores de las unidades asistenciales.

También se requiere el diseño de un plan de acción para la implantación de las recomendaciones y descripción de las estrategias a seguir que debe ser revisado periódicamente y actualizado. Se deben operativizar las recomendaciones de las GBP, con la actualización de los protocolos existentes y la elaboración de otros nuevos, difusión de la información de los nuevos procedimientos, monitorización y evaluación del desarrollo a través de indicadores previamente definidos, adaptación de los sistemas de registros, elaboración de informes, difusión de resultados $\mathrm{y}$, por último, elaboración de un plan de sostenibilidad (4).

En el HRUM se desarrolla esta metodología de implantación de la estrategia que se describe detalladamente a continuación:

- Equipos de implantación: selección de los profesionales -impulsores- para cada una de las GBPs y de cada una de las unidades. Estos impulsores asistenciales tendrán un rol de liderazgo, motivacional y de formación de sus compañeros en las unidades asistenciales.

- Formación: la formación es clave durante todo el proceso. Comienza con la formación de los impulsores en la herramienta de implantación de buenas prácticas de la RNAO (3), para capacitarlos y otorgarles competencias para desarrollar la estrategia del cambio. Estos materiales están definidos por la RNAO. La formación después se concreta en actividades formativas específicas sobre las recomendaciones basadas en las evidencias de cada una de las GBP que se van a implantar $(21,22)$.

- Actualización de protocolos: esta fase permite analizar las recomendaciones en profundidad de las GBP y actualizar en las que se hayan producido evidencias posteriores a la publicación de las GBP.

- Difusión: la difusión comienza con la información de la iniciativa en el centro para que llegue a la mayor cantidad de profesionales. Se pretende la captación de impulsores al programa, pero también informar a los profesionales y las partes interesadas y solicitar su colaboración. También se desarrolla una estrategia para difundir los resultados que se vayan obteniendo del programa. Como novedad, hemos utilizado las redes sociales (especialmente Twitter) como herramienta de difusión de recomendaciones, cuyos resultados expondremos posteriormente.

- Evaluación: la evaluación permite conocer el impacto de la implantación de la estrategia a través de indicadores de procesos y de resultados de salud. 


\section{Resultados de la implementación de la estrategia}

Creación de los equipos de implantación: se han constituido diferentes comisiones de trabajo para llevar a cabo la estrategia (Figura 1):

Figura 1. Diferentes comisiones y equipos de implantación.

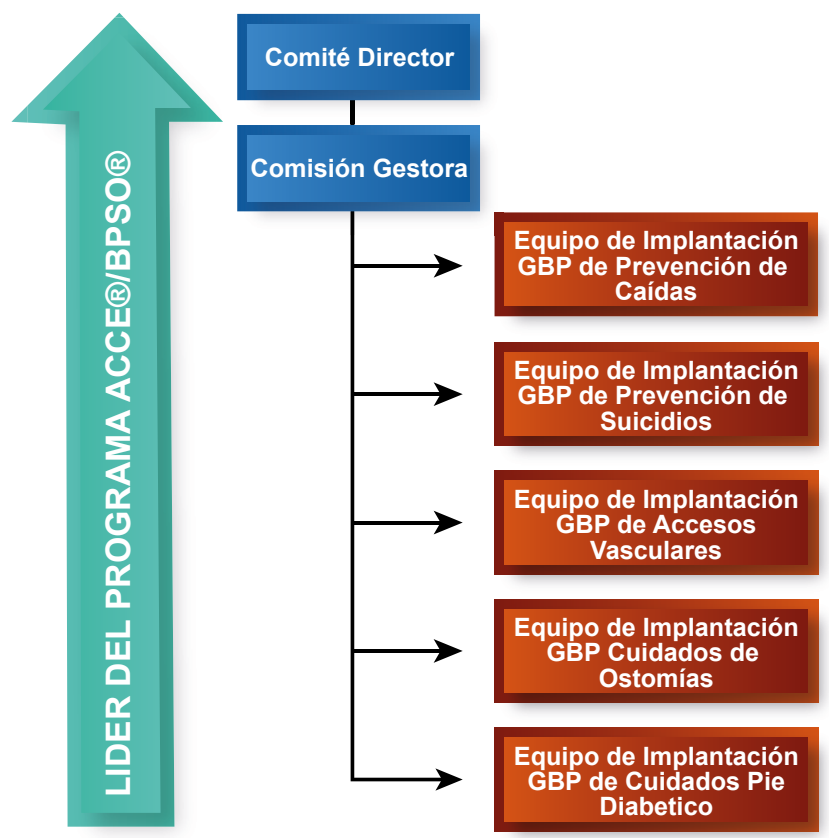

Fuente: Elaboración propia.

- Un comité director: formado por el equipo de dirección de enfermería y el líder del programa en el centro sanitario.

- Una comisión gestora: formada por veinte líderes de la organización pertenecientes a diferentes ámbitos. Esta comisión coordina, dirige y ejecuta la planificación del programa CCEC en el centro sanitario. Tiene funciones asesoras y de participación activa en la planificación y ejecución de las actividades.

- Cinco equipos de implantación: un equipo para cada una de las GBPs a implantar, formado por líderes asistenciales de cada una de las unidades en las que se van a implantar las guías: enfermeras, técnicos en cuidados auxiliares de enfermería, médicos, fisioterapeutas, terapeutas ocupacionales, trabajadores sociales.
Formación: se ha brindado formación a más de 180 impulsores de diferentes profesionales de la salud a través de un curso acreditado por la Agencia de Calidad Sanitaria de Andalucía (ACSA). Paralelamente, se han realizado talleres y sesiones clínicas de cuidados sobre las recomendaciones de las GBPs.

Actualización de protocolos: se han actualizado cuatro procedimientos de trabajo relacionados con las GBP: el protocolo de prevención de caídas, de prevención del suicidio, el cuidado de los catéteres venosos periféricos, el cuidado del pie diabético, y en fase de actualización el cuidado de los catéteres de catéter venoso central y el cuidado de la ostomía.

Difusión: se ha desarrollado un ambicioso plan de difusión para informar a todos los profesionales a través de comisiones clínicas hospitalarias, sesiones informativas en las unidades asistenciales y en la página web del hospital (http://www.hospitalregionaldemalaga. es/Inicio/ExcelenciaenCuidados.aspx)

Una de las actividades muy destacadas y realizadas por los propios profesionales de los equipos de implantación ha sido el diseño de una cartelería específica (tamaño A-2) de cada una de las GBP, con todas las recomendaciones de cuidados basados en las evidencias y que se ha colocado en todas las unidades implantadoras.

Evaluación: la metodología de implantación de evidencias requiere monitorizar los resultados para conocer la evolución y el impacto de la estrategia que se está implantando. RNAO tiene un sistema informático propio denominado CAREVID (NQuIRE), en el que se introduce toda la información extraída de la historia clínica electrónica para evaluar los indicadores de proceso y resultado establecidos en el programa, y que son los mismos en todos los centros sanitarios a nivel mundial.

\section{El uso de las redes sociales como estrategia de difusión}

El uso y la difusión de las recomendaciones a través de las redes sociales, sin duda, ha sido la estrategia que más impacto ha tenido entre los profesionales, constituyendo un elemento innovador y de movilización social. El uso, tanto del Twitter como del Facebook, con nuestros perfiles@EnfermHRUM_BPSO (Twitter) y EnfermHospitalariaHRM (en Facebook), han sido elementos claves que han permitido hacer más visible 
la labor de las enfermeras y potenciar la difusión de las recomendaciones.

Estos perfiles comenzaron a utilizarse desde el comienzo del programa (enero de 2018) con el objetivo de ir difundiendo los avances del mismo. Es en mayo de 2018 cuando introducimos el hashtag \#cuidoenregional, y en agosto de 2018 se diseñó y desarrolló un proyecto específico para difundir las recomendaciones, denominado "CadaSemanaUnaRecomendación", que consiste en que cada semana analizamos cada una de las recomendaciones de una de las GBPs, se actualiza la evidencia, si se precisa, y se especifica cómo se está implantando en el centro y qué actuaciones se están llevando a cabo. Para la difusión semanal se utilizan dos herramientas: el diseño de un folleto semanal y la creación de un video explicativo (el video se realiza cada dos o tres semanas). Para este proyecto semanal se utiliza el hashtag: \#CadaSemanaUnaRecomendación.
Se esponen aquí algunos de los folletos que se han diseñado en las 20 semanas que lleva funcionado el proyecto (Figuras 2-4).

Una de las características que permite Twitter es la identificación de personas, instituciones $u$ organizaciones en cada uno de los tweets que se realizan. Esta posibilidad permite cada semana identificar a personas $\mathrm{u}$ organizaciones relevantes relacionadas con la recomendación, lo que promueve de forma importante su difusión. Se ha conseguido una importante movilización social de los profesionales del centro, ya que cada semana, además de participar en la elaboración del folleto (que permite profundizar en la recomendación), se involucran en el uso de las redes sociales como herramienta de difusión de recomendaciones.

Con respecto a los videos, se hicieron siete videos de difusión de las recomendaciones que se han

Figura 2. Recomendación de la cuarta semana de la GBP de Cuidados y mantenimiento de los accesos vasculares para reducir las complicaciones.

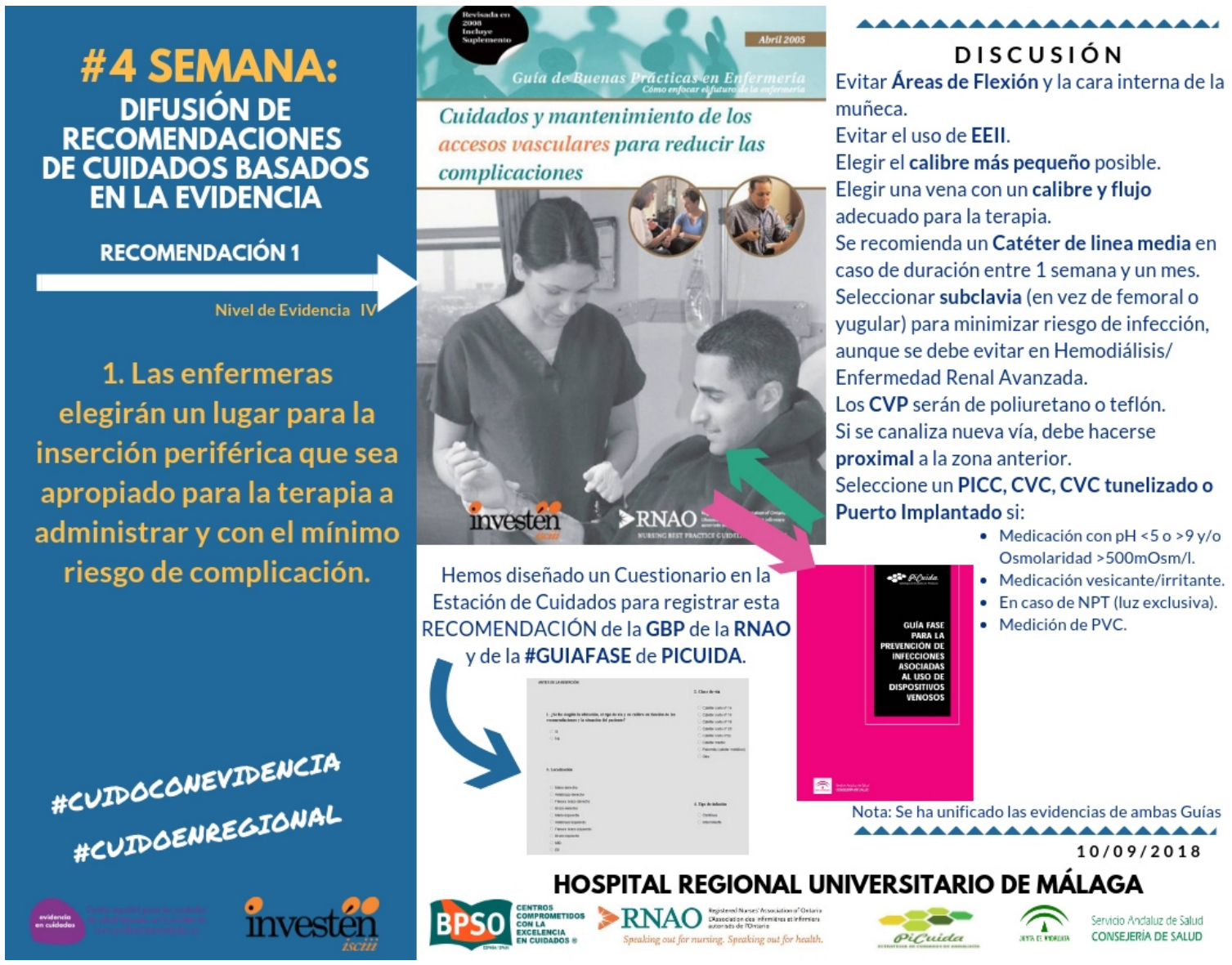

Fuente. Elaboración propia. 
Figura 3. recomendación de la sexta semana de la GBP de Valoración y manejo de las úlceras de pie diabético.

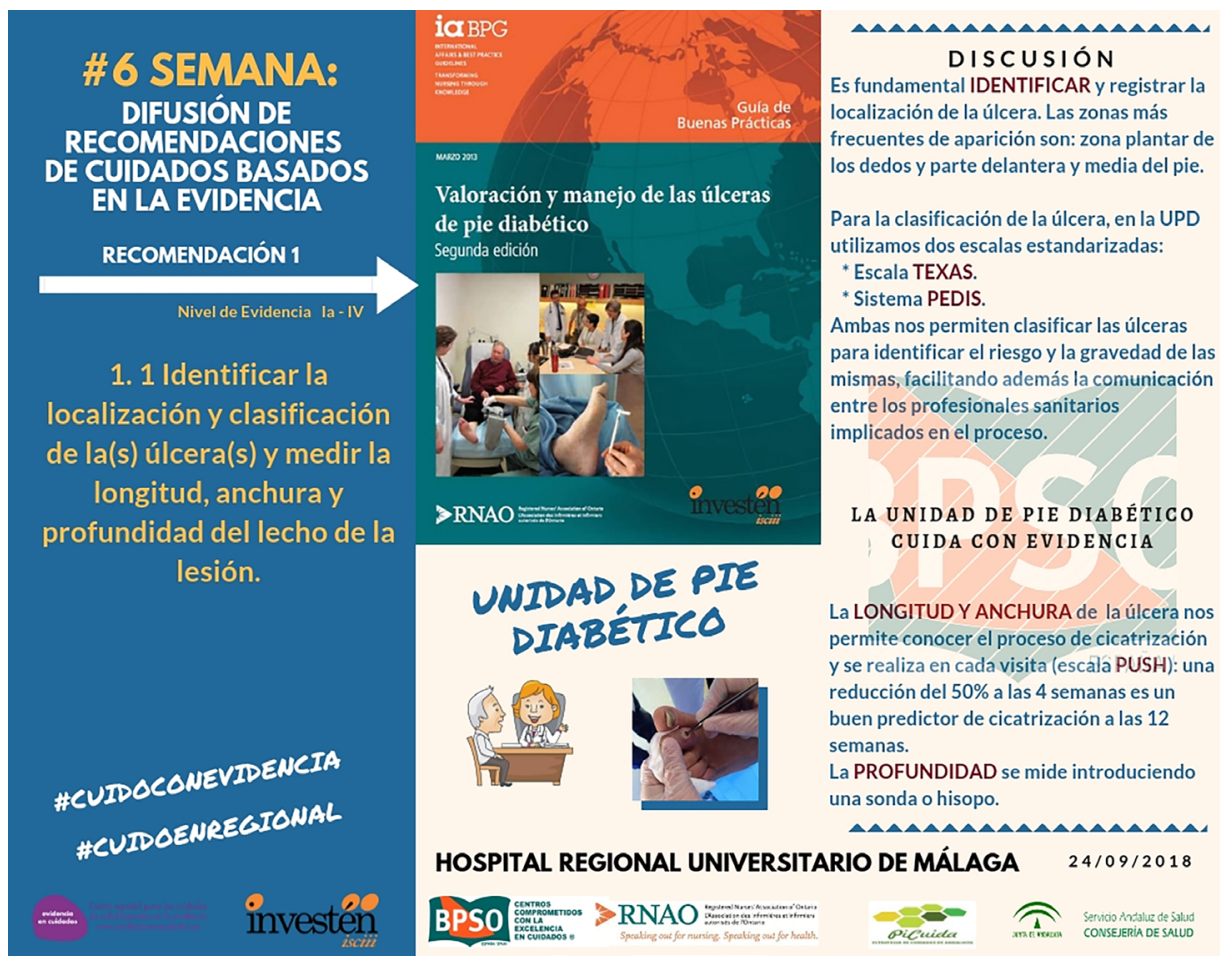

Fuente. Elaboración propia.

Figura 4. Recomendación de la décimotercera semana de la GBP Prevención de caídas y lesiones derivadas de las caídas.

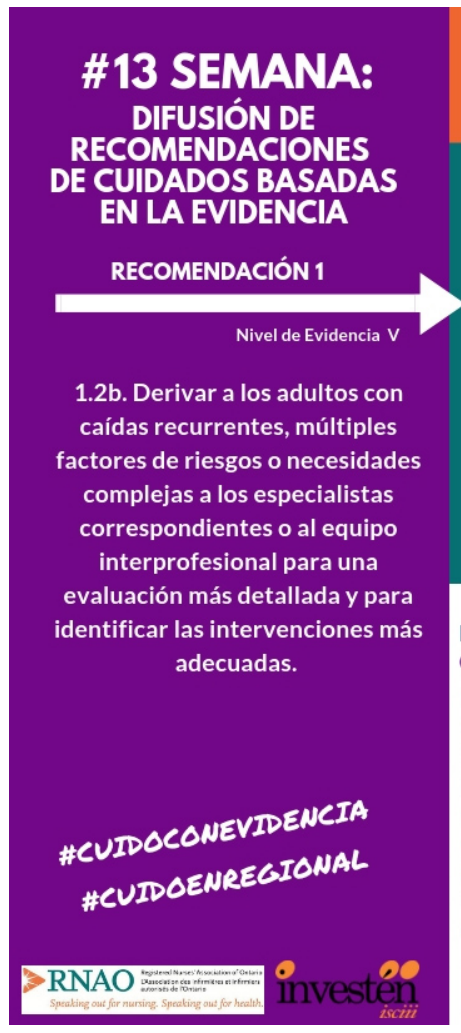

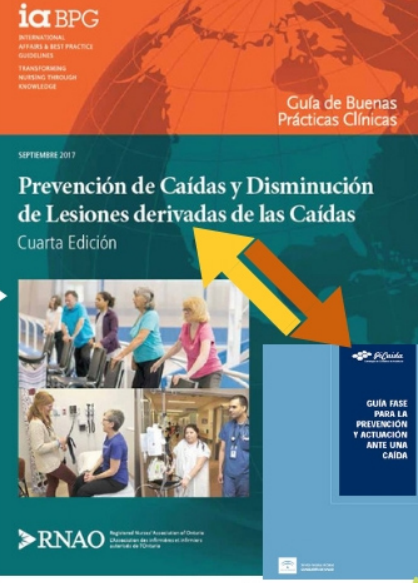

\section{DISCUSIÓN}

Esta recomendación ha sido realizada por el Comité de Expertos de la RNAO (nivel V): La prevención de las caídas debe ser abordada por un equipo multidisciplinar. Todos los profesionales DEBEMOS implicarnos en la prevención de caídas: enfermería, enfermeras, médicos, enfermera sino que debe ser entendida dentro de un gestora de casos, fisioterapeutas, podólogos, Plan Multifactorial de Prevención.

nutricionistas, etc., cada uno desde su competencia profesional.

HOSPITAL REGIONAL UNIVERSITARIO DE MÁLAGA

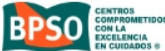

DURANTE LA HOSPITALIZACIÓN

Tras un Cribado Positivo y después de realizar la Valoración Multifactorial para detectar los factores de riesgos, puede ser necesario derivar al paciente a otro profesional sanitario para tratar esos factores de riesgos.

¿QUÉ PROFESIONALES PUEDEN SER NECESARIOS?

Neurológo: en a teraciones del equilibrio o de la marcha que puedan ser causados por el sistema somatosensorial o vestibular.

- Oftalmólogo: en déficits visuales

- Nutricionista: en déficits nutricionales.

Fisioterapeuta: para mejorar el

fortalecimiento muscular.

- Podólogo: cuidado de los pies y calzado.

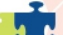

\section{\#CUIDO CON} EVIDENCIA

La coordinación asistencial entre los profesionales de los diferentes ámbito asistenciales (AP $\longrightarrow$ AH) es clave. obstante, la derivación a otro profesional no

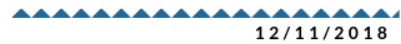

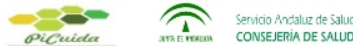

Fuente. Elaboración propia. 
considerado más relevantes. Tanto el diseño de los folletos como los videos, se realizan con la participación de los profesionales que pertenecen a los equipos de implantación, y los folletos cada semana se imprimen y se envían a las unidades asistenciales para que los coloquen en el estar de personal de los profesionales.

Además del hashtag \#CadaSemanaUnaRecomendación, en Twitter utilizamos el hashtag \#cuidoEnRegional (en referencia al nombre de nuestro centro), y que llevamos utilizando desde abril de 2018. Este hashtag se registró en la web Symplur (https://www.symplur.com/), que permite mes a mes hacer una monitorización del uso del hashtag, tanto de los participantes como de las impresiones, y que cada mes se publica en nuestro perfil (Figura 5).

En la Tabla 1 se puede observar el incremento importante de usuarios alcanzados desde que el 20 de agosto de 2018 se comenzó el proyecto

Figura 5. Uso del hashtag \#cuidoenregional desde el 4 de septiembre al 3 de octubre de 2018.

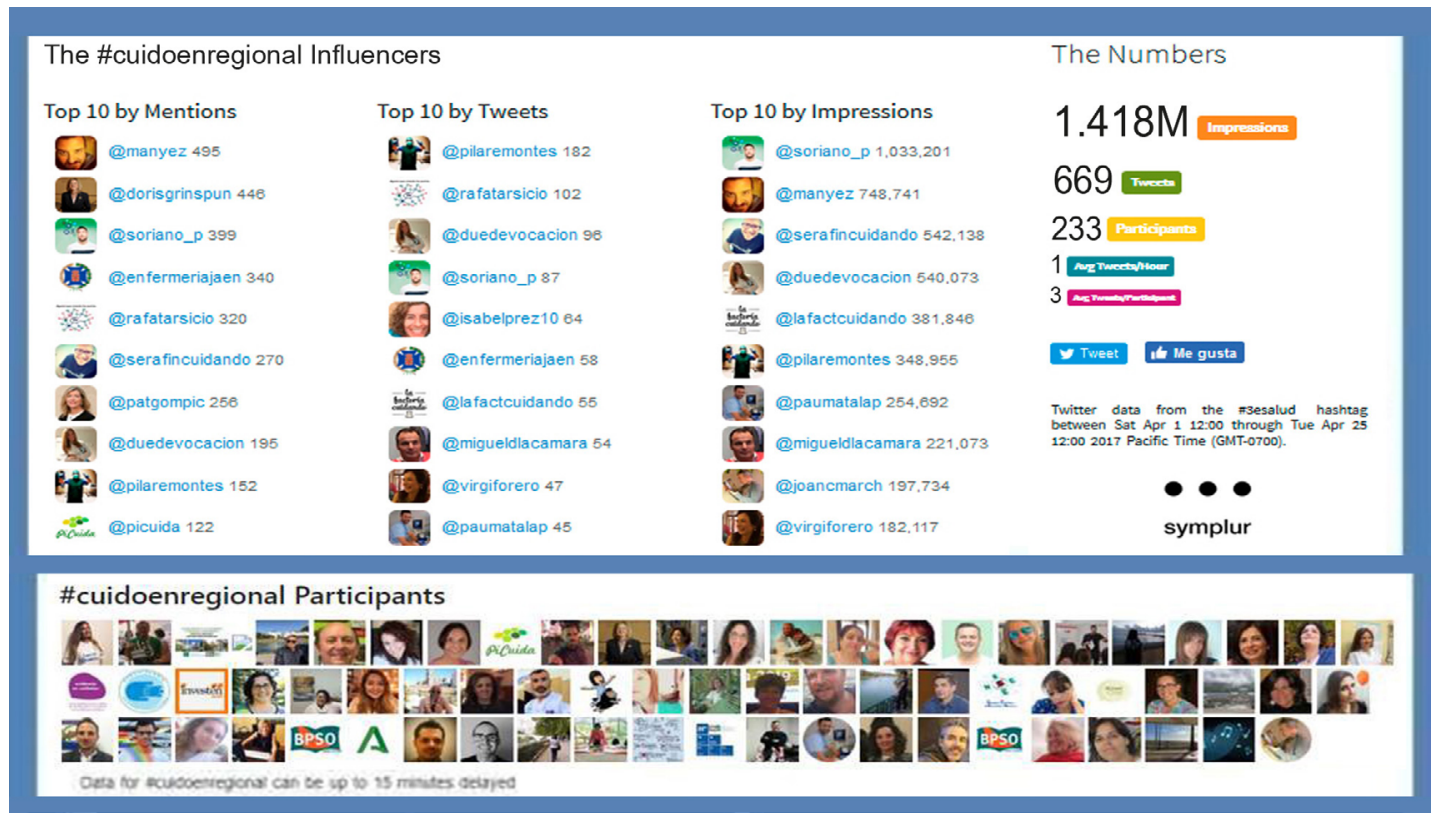

Fuente. Elaboración propia vía @symplur

Tabla 1. Uso del hashtag \#cuidoenregional desde julio de 2018 a diciembre de 2018 (via @symplur).

\begin{tabular}{cccc} 
Meses & Participantes & $\begin{array}{c}\text { Número } \\
\text { de tweets }\end{array}$ & Impresiones \\
\hline $\begin{array}{c}\text { Julio } \\
\text { de } 2018\end{array}$ & 91 & 293 & 444,730 \\
\hline $\begin{array}{c}\text { Agosto } \\
\text { de } 2018\end{array}$ & 82 & 381 & 574,603 \\
\hline $\begin{array}{c}\text { Septiembre } \\
\text { de } 2018\end{array}$ & 233 & 669 & $1,418,000$ \\
\hline $\begin{array}{c}\text { Octubre } \\
\text { de } 2018\end{array}$ & 223 & 588 & $1,029,000$ \\
\hline $\begin{array}{c}\text { Noviembre } \\
\text { de } 2018\end{array}$ & 250 & 655 & $1,152,000$ \\
\hline $\begin{array}{c}\text { Diciembre } \\
\text { de } 2018\end{array}$ & 141 & 486 & 911,618 \\
\hline
\end{tabular}

Fuente. Elaboración propia.
"CadaSemanaUnaRecomendación", así como algunos de los datos que se disponen sobre el incremento cuantitativo del uso del hashtag \#CuidoEnRegional.

Un gran resultado y una experiencia muy positiva ha sido el uso de las redes sociales (especialmente Twitter) para la difusión y la contribución al desarrollo de las recomendaciones de cuidados por varios motivos:

- En primer lugar, en cuanto a una mayor visibilidad de los cuidados, de los profesionales y de nuestro centro. Cada semana, al publicar una recomendación en Twitter, hemos identificado a instituciones nacionales e internacionales relacionadas con el programa (@RNAO,@PICUIDA,@BPSOSpain, Consejería de Salud-@saluland-, @Investeniscii, (a)CCEIJB, etc.), otras veces a sociedades científicas o grupos de investigación (grupo multidisciplinar de acceso vascular -@grumav-, @ProyectoPrebacp, etc.), otras a asociaciones de pacientes (@diabtetesmalaga,@ostomizadosESP, 
etc.) e incluso a profesionales sanitarios y pacientes de referencia a nivel nacional (@duedevocacion, @uciero,@IanBlanco7, @donsacarino, etc.). Esta identificación ha permitido la difusión de las evidencias a través de los re-tweets de estos profesionales o instituciones, proporcionando una mayor visibilidad de los profesionales y de los cuidados que proporcionamos.

- En segundo lugar, se destaca la posibilidad de enriquecer la información con las opiniones y experiencias de otros centros: las redes sociales son una herramienta dinámica y totalmente instantánea. La identificación de personas, profesionales e instituciones en los tweets con las recomendaciones de cuidados ha permitido generar debates clínicos, conocer las experiencias con el uso de otros procedimientos (por ejemplo, otras escalas de valoración del pie diabético o el debate clínico que se generó sobre la osmolaridad en la administración de los medicamentos por vía intravenosa).

- La movilización social de los profesionales: sin duda el uso de Twitter para la implantación de las evidencias ha promovido a los profesionales al uso de la red y ha ayudado en la implantación de las recomendaciones. El hecho de que participen en la elaboración del folleto semanal y que participen en los videos realizados ha supuesto una movilización de sus compañeros para mejorar los cuidados. Son ellos los verdaderos líderes del programa que movilizan a sus compañeros para la realización de las mejores prácticas a través de este movimiento social. Como puede apreciarse en los datos cuantitativos, se duplicó el número de tweets, de participantes y de impresiones de los tweets en el momento en que comenzamos la implantación del proyecto "CadaSemanaUnaRecomendación".

- El liderazgo y desarrollo profesional: nada de esto hubiera sido posible sin la ayuda y colaboración de los líderes de guía y de los profesionales líderes asistenciales de los equipos de implantación que consideran importante este proyecto de "CadaSemanaUnaRecomendación", que han trabajado con gran liderazgo en el desarrollo de la recomendación y el análisis de cómo se están implantando en el centro.

Aunque se han encontrado barreras en el uso de las redes sociales (1) como la falta de tiempo, disminución en la dedicación del tiempo para el cuidado del paciente, disminución de la productividad, falta de tiempo para la formación en el uso de las redes sociales y sobrecarga tecnológica, entre otras, al comparar los resultados con otros estudios, estas pueden ser útiles para la difusión de recomendaciones y de conocimiento.

El desarrollo profesional y la creación de redes van de la mano en las redes sociales.

La creación de redes y la conexión con otros médicos en las redes sociales es uno de los principales beneficios de participación (1).

Al comparar nuestros resultados con otros estudios que también utilizan las redes sociales para la difusión y el debate clínico, nos encontramos con diferentes revistas científicas que utilizan una estrategia de difusión en redes sociales $(23,24)$.

La creación de redes con otros profesionales de la salud en las reuniones por medio de Twitter ahora es la corriente principal. En los últimos años, el uso de Twitter en la reunión anual de la Journal of Clinical Oncology (@ JCO ASCO) ha crecido significativamente $(1,25,26)$.

Aunque en nuestro estudio, los resultados cuantitativos y cualitativos son prometedores, se necesita más tiempo para poder monitorizar el uso de las redes sociales y su posible impacto en la adherencia de la implantación de las recomendaciones de las GBP por los profesionales.

Finalmente, queremos destacar el desarrollo y la implantación de los nuevos registros en los sistemas de registros electrónicos del hospital. Ya estamos teniendo los primeros resultados clínicos basales de los indicadores de las guías, que nos permitirán monitorizar la evolución de los resultados de salud de los pacientes y el posible impacto de la implantación de las recomendaciones en la práctica clínica. Esperamos en breve poder disponer de resultados y publicarlos.

\section{Conclusiones}

Este artículo describe las diferentes actividades realizadas dentro de la estrategia desarrollada en el Hospital Regional Universitario de Málaga para implantar las recomendaciones de cuidados las GBPs de la RNAO en la práctica clínica, y reflexiona en torno a la utilidad de las redes sociales en la difusión de dichas recomendaciones.

En el desarrollo de la estrategia de implantación del modelo, se ha evidenciado un alto nivel de implicación de los profesionales de enfermería en la estrategia. Esto se refleja en una gran participación de los profesionales 
en la formación y en la constitución de los equipos de implantación. Así mismo, la alta implicación se visualiza en la gran motivación y entusiasmo de los profesionales que se manifiesta en un alto nivel de pertenencia al programa y a la implantación de las recomendaciones. Su participación ha sido fundamental en el desarrollo de la estrategia, colaborando en el diseño de la cartelería, con las recomendaciones, actualización de los protocolos y diseño y uso de los cuestionarios para la elaboración de evaluación de los indicadores, así como las diferentes estrategias de difusión basadas en redes sociales, particularmente Twitter.

Entre las estrategias claves para implantar el modelo CCEC en un centro sanitario destaca la formación, el desarrollo de los equipos de implantación y el diseño y uso de nuevos registros en la historia clínica electrónica.

Así mismo, la difusión de las recomendaciones basadas en las evidencias de las GBP de la RNAO a través de las redes sociales es un facilitador y ayuda a la movilización social de los profesionales, y aumenta su nivel de implicación y participación en el programa, aunque se necesita más tiempo para seguir la monitorización del uso de las redes sociales y la evaluación del posible impacto en la adherencia de la implantación de las recomendaciones de las GBP por los profesionales.

La disponibilidad y uso de los registros en la historia clínica nos permitirá conocer el impacto de la implantación de las GBP en la práctica clínica, la adherencia de los profesionales y los resultados de salud de los pacientes.

\section{Agradecimientos}

El presente trabajo ha sido realizado utilizando parcialmente datos y la metodología del programa de implantación de guías de buenas prácticas en Centros Comprometidos con la Excelencia en Cuidados. El autor expresa su agradecimiento al grupo de trabajo del Programa de Implantación de Buenas Prácticas en Centros Comprometidos con la Excelencia en Cuidados.

El programa Centros Comprometidos con la Excelencia en Cuidados está parcialmente financiado por el Centro Español para los Cuidados de Salud basados en la Evidencia: un Centro de Excelencia del Instituto Joanna Briggs.

Agradecemos a todas las enfermeras del HRUM por su participación en el programa de CCEC, por su liderazgo y compromiso con la evidencia.

\section{Referencias}

1. Markham MJ, Gentile D, Graham DL. Social media for networking, professional development, and patient engagement. Am Soc Clin Oncol Educ Book. 2017;37:782-787. Doi: 10.14694/EDBK_180077.

2. Hitlin P. Internet, social media use and device ownership in U.S. have plateaued after years of growth. Pew Research Centre. 2018. Disponible en: http://www.pewresearch.org/fact-tank/2018/09/28/ internet-social-media-use-and-device-ownership-inu-s-have-plateaued-after-years-of-growth/

3. Smith A, Anderson M. A majority of Americans use facebook and youtube, but young adults are especially heavy users of snapchat and instagram. Pew Research Centre. 2018. Disponible en: http:// www.pewinternet.org/2018/03/01/social-media-usein-2018/

4. Albornos L, González E, Moreno T. Implantación de guías de buenas prácticas en España. Programa de centros comprometidos con la excelencia en cuidados. MedUNAB. 2015;17(3):163-169.

5. Registered Nurses Association of Ontario (RNAO). Ontario. International Affair \& Best Practice Guidelines. Disponible en: https://rnao.ca/bpg

6. Registered Nurses Association of Ontario (RNAO). Toolkit: Implementation of best practice guidelines. (2nd ed.). Toronto, Ontario. 2012. Disponible en: http://rnao.ca/sites/rnaoca/files/Toolkit en15.pdf

7. Registered Nurses Association of Ontario (RNAO). Cuidados y mantenimiento de los accesos vasculares. Toronto, Ontario. 2008. Disponible en: http://rnao.ca/ sites/rnao-ca/files/2014_CuidadoAccesoVascular spp 022014.pdf

8. Registered Nurses Association ofOntario. Prevención de caídas y disminución de las lesiones derivadas de las caídas. Toronto, Ontario. 2018. Disponible en: https://rnao.ca/bpg/language/prevenci\%C3\%B3nde-ca\%C3\%ADdas-y-reducci\%C3\%B3n-delesiones-derivadas-de-las-ca $\% \mathrm{C} 3 \% \mathrm{ADdas}$-cuartaedici $\% \mathrm{C} 3 \% \mathrm{~B} 3 n$

9. Registered Nurses Association of Ontario (RNAO). Cuidado y manejo de la Ostomía. Toronto, Ontario. 2009. Disponible en: https://rnao.ca/sites/rnao-ca/ files/BPG_Cuidado_y manejo_de la_Ostomia Guia de buenas practicas en enfermeria.pdf

10. Registered Nurses Association of Ontario (RNAO). Valoración y manejo de las úlceras de pie diabético. Toronto, Ontario. 2013. Disponible en: https://rnao.ca/sites/rnao-ca/files/2015 _ BPG Foot_16 01_2015 - 2nd Edition.pdf

11. Registered Nurses Association of Ontario (RNAO). Toronto, Ontario. 2009. Disponible en: https://rnao. 
ca/bpg/language/valoraci\%C3\%B3n-y-cuidadode-los-adultos-en-riesgo-de-ideaci\%C3\%B3n-ycomportamiento-suicida

12. Grdisa V, Grinspun D, Toor GK, Owusu Y, Naik S et Smith K. Evaluating BPG impact: development and refinement of NQuIRE. En D Grinspun and I Banjnok (Eds). Transforming Nursing Throuhg Knwoledge: Best Practices for Guideline Development, Implementation science, and evaluation. Indianapolis, IN, USA: Sigma Theta Tau International Honor Society of Nursing; 2018. p. 395-431.

13. Grinspun D, Loyd M, Xiao S, Banjnok I. Measuring the quality of evidence-based nursing care: NQuIRE - Nursing Quality Indicators for reporting and Evaluation Data-system. MedUNAB. 2015;17(3):170175. DOI: https://doi.org/10.29375/01237047.2384

14. Van De Velde-Coke S, Doran D, Grinspun D, Hayes L, Boal A-S, Velji K, White P et al. Measuring outcomes of nursing care, improving the health of Canadians: NNQR(C), C-HOBIC, and NQuIRE. Canadian Journal of Nursing Leadership. 2012;25(2):26-37.

15. Aiken LH, Clarke SP, Sloane DM, Lake ET, Cheney T. Effects of hospital care environment on patient mortality and nurse outcomes. J Nurs Adm. 2008;38(5):223-9.

16. Doran D, Lefebre N, O'Brien-Pallas L, Estabrook CA, White P, Carryer $\mathrm{J}$ et al. The relationship among evidence-based practice and client dyspnea, pain, falls, and pressure ulcer outcomes in the community setting. Worldviews Evid Based Nurs. 2014;11(5):274-83.

17. Pericas J, González S, De Pedro J, Morales JM, Bennasar M. Perception of Spanish primary healthcare nurses about evidence-based clinical practice: a qualitative study. Int Nurs Rev. 2014;61(1):90-8.

18. Grol R, Grimshaw J. From best evidence to best practice: effective implementation of change in patients' care. Lancet Lond Engl. 2003;362(9391):1225-30.

19. Beard E. Nurse Leaders and the use of social media: becoming literate to lead. Nursing Leader; 2013; 11(6): 38-40. Disponible en: https://doi. org/10.1016/j.mnl.2013.09.002

20. Surani Z, Hirani R, Elias A, Quisenberry L, Varon J, Surani $S$ et al. Social media usage among health care providers. BMC Res Notes. 2017; 10:654. Doi:10.1186/s13104-017-2993-y

21. Grinspun, D. Transforming Nursing Through Knowledge: The conceptual and programmatic underpinnings of RNAO's BPG Program. En D. Grinspun and I. Bajnok (Eds.), Transforming Nursing Through Knowledge: Best Practices for Guideline Development, Implementation Science, and Evaluation. Indianapolis, IN, USA: Sigma Theta Tau International Honor Society of Nursing; 2018. p. 3-27.
22. Bajnok I, Grinspun D, Grdisa V. RNAO's Global Spread of BPGs: The BPSO Designation Sustainability and Fidelity. En D. Grinspun and I. Bajnok (Eds.), Transforming Nursing Through Knowledge: Best Practices for Guideline Development, Implementation Science, and Evaluation. Indianapolis, IN, USA: Sigma Theta Tau International Honor Society of Nursing; 2018. p. 287-317.

23. Roberts MJ, Perera M, Lawrentschuk N, et al. Globalization of continuing professional development by journal clubs via microblogging: a systematic review. J Med Internet Res. 2015;17:e103.

24. Thangasamy IA, Leveridge M, Davies BJ, et al. International Urology Journal Club via Twitter: 12-month experience. Eur Urol. 2014;66:112-117.

25. Chaudhry A, Glodé LM, Gillman M, et al. Trends in twitter use by physicians at the American Society of Clinical Oncology Annual Meeting, 2010 and 2011. J Oncol Pract. 2012;8:173-178.

26. Katz M. "Twitter Use at Three Annual Professional Meetings (2012-2014)". 2014 